\title{
Electrode Reactions of Organic Compounds GENERAL INTRODUCTION
}

\author{
By R. A. Marcus \\ Noyes Chemical Laboratory, University of Illinois, Urbana, \\ Illinois 61801, U.S.A.
}

Received 4th June, 1968

\section{INTRODUCTION AND SURVEY}

The last Faraday Society Discussions on Electrode Processes were held in 1947 and marked the onset of an extensive fruitful post-war development of fast reaction techniques in electrochemistry. Considerable information has since been obtained on rate constants of simple inorganic electron transfers, paralleling a similar development in homogeneous reactions, and on electron transfer theory. A theoreticallybased relation exists between the two areas.

It has often been noted that the Faraday Society Discussions have marked a turning point in a field. The area of organic reactions at electrodes has been somewhat on the periphery of the field of organic chemistry, its relation to the subject of metal-catalyzed organic syntheses notwithstanding. The papers of the present Discussions illustrate, perhaps for the first time as a body, the rich variety of studies in the present field and a role for current organic concepts.

The papers may be loosely classified into the following overlapping categories.

PRIMARILY THEORETICAL.-Hoytink, ${ }^{1}$ on energetics of reactions of aromatic ions leading to excited states and applications of electron transfer theory; Mehl and Hale, on reactions at the interface of an anthracene electrode and an electrolyte and applications of the same electron transfer theory; Hush and Segal, on the stability of $\mathrm{CH}_{3} \mathrm{~F}^{-}$ relative to $\mathrm{CH}_{3} \mathrm{~F}$ and possible implications for electron transfer with bond fission; and Parsons, on the role of adsorption on reaction schemes and current-potential plots. Other papers employ several current theoretical concepts of physical-organic chemistry.

ADSORPTION.-Parsons, cited earlier; Brummer and Cahill, on interaction of $\mathrm{H}$ atoms with adsorbed species. Breiter, on anodic oxidation of $\mathrm{CH}_{3} \mathrm{OH}$; Conway et $a l$, on reaction order, $i-V$ plots and kinetic isotope effect for acetophenone reduction; Brown and Lister, on reaction order in acetone reduction; and Hickling and Wilkins, on inhibition of $\mathrm{O}_{2}$ evolution by adsorbed species for Kolbe and other reactions.

CARBONYL REDUCTION.-Conway et al., steady-state studies, just cited ; Brown and Lister, just cited, and hydrocoupling with acrylonitrile, study of intermediates via potential switching; and Zuman et al., on polarography of unsaturated ketones and of alphatic aldehydes.

OTHER REDUCTIONS.-Lund, on polarographic reduction of heterocyclics; Gygax and Jordan, on polarographic and controlled potential electrolysis (c.p.e.) of haemoprotein models; and Littlehailes and Woodhall, on study of electrochemically and chemically prepared quaternary ammonium-sodium amalgams used in reductions. 
STEREOCHEMISTRY IN ELECTROCHEMISTRY.-Grabowski et al., on least structural change rule and electrode reactions, including inversion or retention of configuration in $\mathrm{RCl}$ reductions; Dietz and Peover, on effect of planarity on reduction rates of cis and trans substituted stilbenes and on related systems; and Bard et al., on comparison of polarographic reduction potentials, spin resonance data and m.o. calculations for sterically hindered substituted anthracenes.

ANODIC OXIDATION.-Kuwana and Strojek, on spectral study (with opticallytransparent electrodes), cyclic voltammetry and c.p.e. for the oxidation of o-toluidine ; Eberson and Nilsson, on anodic cyanation and similarities to data in homogeneous electrophilic substitutions; Tsutsumi and Koyama, on differences in anodic cyanation and anodic methylation, and relation to homogeneous relative reaction rates; Fleischman and Goodridge, on effect of square-wave pulses in Kolbe and HoferMoest syntheses ; Hickling and Wilkins, on square wave pulse and potential-switching studies in Brown-Walker and Kolbe syntheses.

\section{THEORETICAL REVIEW. WEAK-OVERLAP ELECTRON TRANSFERS}

In the organic reactions considered in this Discussion there are those for which existing weak-overlap electron transfer theory might suffice, in some cases with due allowance for the role of adsorption, and those (usually atom or proton transfers)

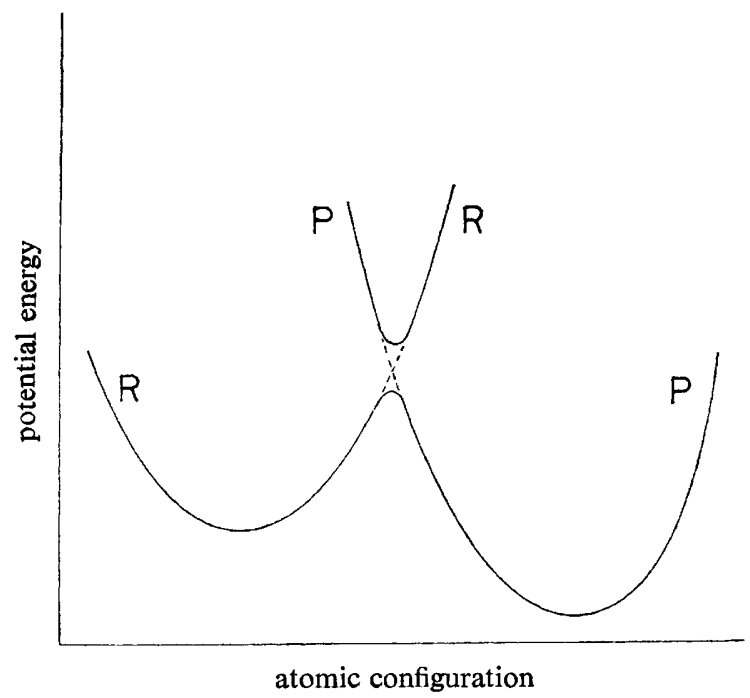

FIG. 1.-Profile of potential energy surface of reactants $(R)$ and that of products $(P)$ plotted against configuration of all atoms in the system, for weak-overlap reactions : - - , surface for zero electronic interaction of the reacting species; - , adiabatic surface. The surfaces are given for some specified separation distance of the two reacting species. The plot for electrode reactions is similar but contains many surfaces (fig. 2, ref. (3)).

in which the electronic interaction of the reactants is large. More specifically, the mechanism in the former case can be described in terms of the properties of two potential energy surfaces, one for the reactants and one for the products. ${ }^{2}$ The weak interaction causes a small splitting in the intersection region (fig. 1). In the second case, the interaction is so large that the potential energy profile now resembles one of the alternatives in fig. 2. It is pertinent, therefore, to review several aspects of these problems. In passing, one notes that the adsorption studies include those 
where the interest is centred on the adsorption phenomenon itself and those designed to find simple conditions for detailed kinetic investigation of reaction mechanisms.

We first recall that for weak-overlap electron transfer reactions the following theoretical relation has been derived ${ }^{3}$ and that there are a number of experimentally interesting consequences. ${ }^{2}{ }^{4}$

$$
\begin{aligned}
k & =\kappa \rho Z \exp \left(-\Delta F^{*} / \boldsymbol{R} T\right), \\
\Delta F^{*} & =w^{\mathrm{r}}+(\lambda / 4)(1+\Delta / \lambda)^{2} .
\end{aligned}
$$

where $\rho$ is of the order of unity; $Z$ is the collison number $\left(\sim 10^{14} \mathrm{~cm}^{3} \mathrm{~mole}^{-1} \mathrm{sec}^{-1}\right.$ for homogeneous reactions and $\sim 10^{4} \mathrm{~cm} \mathrm{sec}^{-1}$ for electrode reactions); $\kappa$ is a transition probability, being unity for quantum mechanically adiabatic reactions; $w^{\mathrm{r}}$ is the work required to transport the reactions (or reactant plus electrode) together ; $\lambda / 4$ is an "intrinsic barrier", related to the barriers of certain isotopic exchange reactions ; $\Delta$ is $\Delta F^{\circ \prime}+w^{\mathrm{p}}-w^{\mathrm{r}}$ for homogeneous reactions and is $n_{\mathrm{e}} F\left(E-E^{\circ \prime}\right)+w^{\mathrm{p}}-w^{\mathrm{r}}$ for electrode reactions, where $n_{\mathrm{e}}$ is the number of electrons transferred.

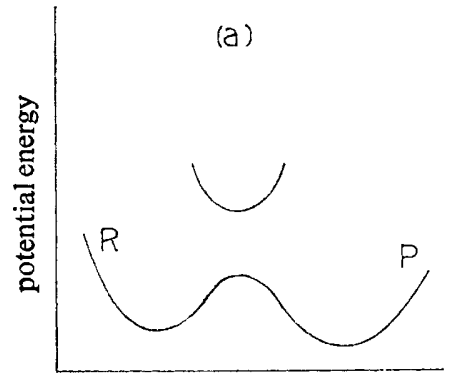

atomic configuration (b)

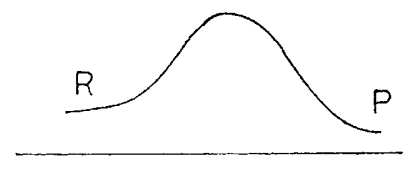

atomic configuration

FIG. 2.-Profile of potential energy surface for strong-overlap reactions, including atom and proton transfers. In system $(a)$ the transfer occurs primarily near some given $\mathbf{A}_{1} \mathrm{~A}_{2}$ separation distance; in $(b)$ there is an appreciable change of $\mathrm{A}_{1} \mathrm{~A}_{2}$ distance during the tra nsfer (appendix II, ref. (10)).

One set of corollaries of the equation arises because of an additivity property of $\lambda$ and permits one to deduce relations such as (2.3) to (2.5) and capable of experimental test $^{2,5}$ :

$$
\begin{aligned}
k_{12} & \cong\left(k_{11} k_{12} K_{12} f_{12}\right)^{\frac{1}{2}}, \\
\left(k_{11} / Z\right)^{\frac{1}{2}} & \cong k_{\mathrm{el}} / Z_{\mathrm{el}}, \\
k_{12} / k_{\mathrm{el}, \mathrm{E}} & \cong \text { constant, }
\end{aligned}
$$

where $k_{12}$ is the homogeneous reaction rate constant for cross-reaction,

$$
\mathrm{A}_{1}^{\mathrm{ox}}+\mathrm{A}_{2}^{\mathrm{red}} \rightarrow \mathrm{A}_{1}^{k_{12}}+\mathrm{A}_{2}^{\mathrm{ox}} \text {. }
$$

$K_{12}$ is its equilibrium constant in the given medium, $k_{i l}$ is the rate constant for the isotopic exchange reaction,

$$
\mathrm{A}_{i}^{\mathrm{ox}}+\mathrm{A}_{i}^{\mathrm{red}} \stackrel{k_{i t}}{\rightarrow} \mathrm{A}_{i}^{\mathrm{red}}+\mathrm{A}_{i}^{\mathrm{ox}}
$$

$f_{12}$ is a known function of $k_{11}, k_{22}, K_{12}$ and arises from the $\Delta^{2} / 4 \lambda$ term in the expansion of (2.2), $k_{\mathrm{e} 1}$ and $k_{\mathrm{el}, \mathrm{E}}$ are the rate constants of $(2.8)$ at $E=E^{\circ}$ and at any fixed $E$, respectively,

$$
\mathrm{A}^{\mathrm{ox}}+\mathrm{M}(\mathrm{e}) \rightarrow \mathrm{A}^{\mathrm{red}}
$$

Conditions for deriving (2.3) to (2.5) from (2.2) are noted in ref. (4). 
From (2.2) one can derive a relation for $\partial \Delta F^{*} / \partial \Delta F^{\circ}$, the Brönsted slope of homogeneous reactions.

$$
\alpha=\frac{1}{2}(1+\Delta / \lambda) \cong \frac{1}{2}\left(1+\Delta F^{\circ \prime} / 4 \Delta F_{0}^{*}\right)
$$

where $\Delta F_{0}^{*}$ is the intercept at $\Delta F^{\circ \prime}=0$ of a plot of $-\boldsymbol{R} T \ln k$ against $\Delta F^{\circ \prime}$. A related expression is obtained for electrode reactions.

Eqn. (2.2), which has a variety of consequences, has been derived with varying degrees of generality. However, underlying each derivation are two principles, which might be termed "conservation" and "quadratification". The former is revealed in various ways. For example, the function $\mathbf{P}_{\mathrm{u}}(\mathbf{r})$, related to the orientation polarization at each point $\mathbf{r}$ in the medium when the system is an activated complex, was shown to be given by ${ }^{6}$

$$
\mathbf{P}_{\mathrm{u}}=\alpha_{\mathbf{u}}\left[(1-n) \mathbf{E}^{\mathrm{r}}+n \mathbf{E}^{\mathfrak{p}}\right]
$$

where $n$ proves to be $\partial \Delta F^{*} / \partial \Delta F^{\circ \prime}$ in the homogeneous system and $\partial \Delta F^{*} / \partial\left[n_{\mathrm{e}} F\left(E-E^{\circ \prime}\right)\right.$ in the electrode reaction one. ( $\mathbf{E}^{\mathbf{r}}$ and $\mathbf{E}^{\mathfrak{p}}$ are electrical fields; $\alpha_{\mathbf{u}}$ is a susceptibility.) Again, a more general (statistical mechanical rather than dielectric continuum) treatment of the environment yields (2.11) for the effective potential energy governing the Boltzmann-weighted configurational distribution of the activated complexes in many-dimensional configuration space, i.e., of systems at the intersection of the two surfaces in fig. $1^{2,7}$ :

$$
U^{\mathbf{e f f}}=(1-n) U^{\mathbf{r}}+n U^{\mathbf{p}} .
$$

$\left[U^{\mathbf{r}}\right.$ and $U^{\mathbf{p}}$ are the potential energies of a system possessing the charge distribution of reactants and that of the products, respectively.] Thus, there is "conservation", in the sense of a " give and take" between the properties of $U^{\mathrm{r}}$ and those of $U^{\mathrm{p}}$, or between $\mathbf{E}^{\mathbf{r}}$ and $\mathbf{E}^{\mathrm{p}}$.

The "quadratification" arises from two of the approximations made in deriving (2.2). One is that the vibrational motion within the co-ordination shells of the reactants is harmonic (or, over the change of distances involved, can be treated as that of an equivalent harmonic oscillator). Thereby, the vibrational potential energy is a quadratic function of fluctuations in vibrational co-ordinates. The second assumption is that the surrounding medium can be treated as dielectrically unsaturated, i.e., that the free energy of the system is a quadratic function of fluctuations in dielectric polarization of the medium.

We note further that for weak-overlap electron transfer reaction the theoretical interpretation for the Brönsted slope, $\partial \Delta F^{*} / \partial \Delta F^{\circ \prime}$, or for its electrochemical counterpart $\partial \Delta F^{*} / \partial\left[n_{\mathrm{e}} F\left(E-E^{\circ \prime}\right)\right]$, is ${ }^{7}$

$$
\alpha=\left\langle\partial U^{\mathbf{r}} / \partial q\right\rangle /\left(\left\langle\partial U^{\mathbf{r}} / \partial q\right\rangle-\left\langle\partial U^{\mathfrak{p}} / \partial q\right\rangle\right)
$$

where $q$ is the reaction co-ordinate and the averages \langle\rangle are over the activated complexes in the many-dimensional configuration space, distributed in a Boltzmann population. $^{8}$

\section{THEORETICAL REVIEW. ATOM AND PROTON TRANSFERS}

A somewhat different situation confronts us for atom transfers, proton transfers, or very-strong-overlap electron transfers. Now, the usual splitting of reactants' and products' potential energy surfaces is so large in the activated complex region that a discussion of mechanisms based on intersecting surfaces and on a calculation of their properties there is no longer meaningful. Thus, quantities such as the slopes $\partial U^{\mathbf{r}} / \partial q$ and $\partial U^{\mathfrak{p}} / \partial q$ are no longer well-defined, and one must seek elsewhere for a 
derivation of a theoretical expression for $\Delta F^{*}$ and for a theoretical interpretation of $\alpha$. The counterpart of reaction (2.6) is now

$$
\mathrm{A}_{1} \mathrm{~B}+\mathrm{A}_{2} \rightarrow \mathrm{A}_{1}+\mathrm{BA}_{2} \text {, }
$$

where $B$ is the atom or proton being transferred. In an electrode reaction, the reaction may involve bond ruptures such as the following:

$$
\begin{aligned}
& \mathrm{AB}+\mathrm{M}(\mathrm{e}) \rightarrow \mathrm{A}+\mathrm{B}^{-}+\mathrm{M} \\
& \mathrm{C}+\mathrm{AB}+\mathrm{M}(\mathrm{e}) \rightarrow \mathrm{CA}+\mathrm{B}^{-}+\mathrm{M} \\
& \mathrm{AB}+\mathrm{M}(\mathrm{e}) \rightarrow \mathrm{A}+\mathrm{BM}(\mathrm{e}) \rightarrow \text { etc. }
\end{aligned}
$$

As noted earlier, the theoretical constructs associated with two intersecting potential energy surfaces should now be replaced by others. At the present time, resort must be made to approximate treatments. (Even the three-electron system, $\mathrm{H}+\mathrm{H}_{2} \rightarrow \mathrm{H}_{2}+\mathrm{H}$, is only now beginning to yield to more exact approaches.) One simple model is the bond-energy bond-order model of $\mathrm{H}$. S. Johnston. ${ }^{9}$ He assumed that the total bond order in (3.1) was constant along the reaction path, ultilized bond order-bond energy relations, found the potential energy maximum along the path, and calculated activation energies using parameters obtained from non-kinetic data. He obtained reasonable agreement with the data.

This model offers a simple method for exploring some of the topics cited earlier. The model obeys, incidentally, the conservation theme described earlier, the " conservation" now being one of a bond order. Recently, we showed ${ }^{10}$ that calculations of potential energy barriers based on the Johnston model are well-approximated by (2.2), even though the potential energy profile along the reaction co-ordinate now has a vastly different shape (fig. $2 b$ ). Correspondingly, for atom transfers, when there are only minor solvation corrections and when the entropy changes cancel or are minor, (2.2), and its consequences such as (2.3) and (2.9) once again obtain. ${ }^{10} .{ }^{11}$ However, theoretical interpretation of the Brönsted slope $\alpha$ is now different. It was shown to be 10

$$
\alpha=n_{\mathrm{BA}_{2}}^{\neq}
$$

where $n_{\mathrm{BA}_{2}}^{ \pm}$is the bond order of the $\mathrm{BA}_{2}$ bond being formed in the activated complex of reaction (3.1). That is, the interpretation of $\alpha$ no longer depends on quantities such as $\partial U^{\mathbf{r}} / \hat{c} q$, which are not meaningful for the present profiles, but is expressed instead in terms of properties of the new model, bond orders in the present instance.

When large solvation changes occur, as in proton transfers, there can be two contributions to the barrier. One involves desolvation of one $A_{i}$ and solvation of the other in reaction (3.1). (Now, B is $\mathrm{H}^{+}$there.) A second involves the barrier, if any, for the gas-phase proton transfer. The treatment of these reactions in solution is currently being explored by us using a combination of a method used for gas-phase proton transfers and one related to that employed for treating solvation in electron transfers. In the meanwhile, (2.2) has been used as a conjecture to analyze data on proton transfers, Brönsted slopes, and kinetic isotope effects. ${ }^{12}$ While the possible applicability of related theoretical considerations to the electrode reactions (3.2)-(3.4) has not yet been explored, we intend to investigate this problem. Experimental kinetic data for various electrode reaction series on these reactions and on analogous homogeneous reactions would provide a useful guide in analyzing this somewhat complex theoretical situation.

I shall conclude this section with an example relating homogeneous and electrochemical results on reduction of acetophenones. ${ }^{13}$ The polarographic wave for 
reduction of substituted acetophenones in high acid media is reversible and is believed to be associated with the reaction,

$$
\mathrm{X}-\mathrm{C}_{6} \mathrm{H}_{4}-\mathrm{CO}-\mathrm{CH}_{3}+\mathrm{H}^{+}+\mathrm{M}(\mathrm{e}) \rightleftharpoons \mathrm{X}-\mathrm{C}_{6} \mathrm{H}_{4}-\mathrm{COH}-\mathrm{CH}_{3}+\mathrm{M} \text {. }
$$

Data are available on the effect of $\mathrm{X}$ on the half-wave potentials, $E_{\frac{1}{2}} .{ }^{14}$ The equilibrium results may be compared with kinetic data ${ }^{15}$ for addition of radicals $R$. (obtained from di-t-butyl peroxide) to substituted acetophenones.

$$
\mathrm{X}-\mathrm{C}_{6} \mathrm{H}_{4}-\mathrm{CO}-\mathrm{CH}_{3}+\mathrm{R} \cdot \stackrel{k}{\rightarrow} \mathrm{X}-\mathrm{C}_{6} \mathrm{H}_{4}-\mathrm{COR}-\mathrm{CH}_{3}
$$

The slope of kinetic plots, $\log k_{\mathrm{x}}$ against $\sigma_{\mathrm{x}}$, where $\sigma_{\mathrm{x}}$ is Hammett's substituent constant for substituent $\mathrm{X}$, is designated by $\rho_{\mathrm{c}}$. The slope of the $E_{\frac{1}{2}}^{\mathrm{x}}$ against $\sigma_{\mathrm{x}}$ plot for reaction (3.6) can be written analogously as $\left(2 \cdot 3 R T / n_{\mathrm{e}} F\right) \rho_{\mathrm{el}}$. (This $\rho_{\mathrm{e} 1}$, which has the same units as $\rho_{\mathrm{c}}$, differs from that defined in ref. (14) by a factor of $2 \cdot 3 R T / n_{\mathrm{e}} F$.) If the principal effect of the $\mathrm{X}$ in reaction (3.7) were to alter the $\Delta F^{\circ}$ for that reaction, changes in $\Delta F^{\circ \prime}$ would be equal to changes in $E_{\frac{1}{2}}$ in (3.6), assuming the difference of chemical potentials of the radicals in the right hand side of (3.6) and (3.7) to be independent of X. Thus, the Brönsted slope for reaction (3.7) can now be written as

$$
\alpha=\frac{\partial \Delta F_{\mathrm{x}}^{*}}{\partial \Delta F_{\mathrm{x}}^{\circ}}=\frac{2 \cdot 3 R T}{n \boldsymbol{F}} \frac{\partial \log k_{\mathrm{x}} / \partial \sigma_{\mathrm{x}}}{\partial E_{\frac{1}{2}}^{\mathrm{x}} / \partial \sigma_{\mathrm{x}}}=\frac{\rho_{\mathrm{c}}}{\rho_{\mathrm{el}}}
$$

$\rho_{\mathrm{c}}$ was found to be $1.59^{15}$ and $\rho_{\mathrm{el}}$ ranges from $3 \cdot 2$ to 4.5 for various media and investigators. ${ }^{14}$ The corresponding values of $\alpha$ thus lie in the range $0 \cdot 50-0 \cdot 35$. It would be desirable, however, to make the comparison of this equilibrium electrochemical and kinetic homogeneous data in a similar solvent medium, and to extend the comparison to other systems.

\section{CONCLUSION}

The papers of the present Discussion illustrate a rich variety of mechanistically interesting reactions in the field of organic reactions at electrodes. Some are presumably weak-overlap electron transfers, for which existing theory may suffice, with detailed analysis of adsorption when needed. Some are atom transfers involving concerted or dissociative bond rupture (i.e., $S_{\mathrm{N}^{2}}$ or $S_{\mathrm{N}^{1}}^{\circ}$, respectively). For such reactions, a different theoretical framework is being constructed and being tested. It has several features in common with the first type of theory, but also has certain differences arising from the different shape of the potential energy profiles in the two cases. A theoretical interpretation of the Brönsted slope, for example, is in one system given by (2.12) and in the other by (3.5). Theory and experiment have much to profit by continuing for these reactions the useful partnership they have enjoyed in the past decade for weak-overlap electron transfers.

${ }^{1}$ in ref. (14) of Hoytink, i.e., in R. A. Marcus, J. Chem. Physics, 1965, 43, 2654, the lower R and $\mathbf{P}$ curves in fig. $1 c$ should have been joined, as should the upper $\mathbf{P}$ and $\mathbf{R}$ curves.

2 R. A. Marcus, Disc. Faraday Soc., 1960, 21, 29.

${ }^{3}$ A theoretical review is given in R. A. Marcus, Ann. Rev. Physic. Chem., 1964, 15, 155, where comparison is made with the interesting results of Levich and Dogonadze, Hush, Gerischer, and other investigators.

${ }^{4}$ R. A. Marcus, J. Physic. Chem., 1963, 67, 853, 2889.

5 for a recent survey of experimental data, see N. Sutin, Ann. Rev. Physic. Chem., 1966, 17, 119.

${ }^{6}$ R. A. Marcus, J. Chem. Physics, 1956, 24, 966 . We have changed the notation somewhat, e.g., $n$ replaces $1+m$. 


\section{R. A. MARCUS}

${ }^{7}$ R. A. Marcus, J. Chem. Physics, 1965, 43, 679.

${ }^{8}$ It follows from (2.12) that when the P surface in fig. 1 intersects the $\mathrm{R}$ surface near the latter's minimum, $\langle\partial U \mathrm{r} / \partial q\rangle$ vanishes and $\alpha$ is zero. When the intersection occurs instead near the minimum of the P surface, $\langle\partial U \mathrm{p} / \partial q\rangle$ vanishes and $\alpha$ is unity. When instead the magnitude of the average slopes in fig. 1 are equal at the intersection, i.e., when $\langle\partial U \mathrm{p} / \partial q\rangle=-\langle\partial U \mathrm{r} / \partial q\rangle$, $\alpha$ equals 0.5 . In these three cases, one sees that the activated complex, which occurs at the intersection of the two surfaces, resembles the reactants, the products, and a complete compromise between the favourable configurations of reactants and products, respectively.

${ }^{9}$ H. S. Johnston, Adv. Chem. Physics, 1960, 3, 131 ; C. Parr and H. S. Johnston, J. Amer. Chem. Soc., 1963, 85, 2544.

${ }^{10}$ R. A. Marcus, J. Physic. Chem., 1968, 72, 891. [see eqn. (6), for the necessary modification of (2.2) prevailing when $|\Delta / \lambda| \geqslant 1$.]

11 A pioneering application of (2.3) to atom transfers in solution was made by N. Sutin in ref. (5), p. 154 and in Proc. Exchange Reactions Symp., (Upton, N.Y., 1965), p. 7, and by A. Haim and N. Sutin, J. Amer. Chem. Soc., 1966, 88, 434.

12 A. O. Cohen and R. A. Marcus, J. Physic. Chem. 1968, 72, 000.

${ }^{13}$ I am indebted to Dr. Frank Ludwig of this laboratory, who selected these reactions and made the necessary theoretical analysis.

${ }^{14}$ P. Zuman, Substituent Effects in Organic Polarography (Plenum Press, New York, 1967), Table III-4, surveys the data of several investigations.

15 E. S. Huyser and D. C. Neckers, J. Amer. Chem. Soc., 1963, 85, 3641. 